\title{
A Novel Hybrid Intelligence Approach for 2D Packing through Internet Crowdsourcing
}

\author{
Anupam Agrawal*, Parmatma Yadav, C. K. Upadhyay \\ Dept. of Information Technology \\ Indian Institute of Information Technology Allahabad, \\ Deoghat, Jhalwa, Allahabad, India. \\ Email:*anupam@iiita.ac.in
}

\begin{abstract}
Packing problems on its current state are being utilized for wide area of industrial applications. The aim of present research is to create and implement an intelligent system that tackles the problem of $2 \mathrm{D}$ packing of objects inside a $2 \mathrm{D}$ container, such that objects do not overlap and the container area is to be maximized. The packing problem becomes easier, when regular/rectangular objects and container are used. In most of the practical situations, the usage of irregular objects comes to existence. To solve the packing problem of irregular objects inside a rectangular container, a hybrid intelligence approach is introduced in our proposed work. The combination of machine intelligence and human intelligence is referred as the hybrid intelligence or semi-automated approach in the proposed methodology. The incorporation of human intelligence in the outcome of machine intelligence is possible to obtain using the internet crowdsourcing as we wish to handle the packing problem through internet crowdsourcing involving rural people. The proposed methodology is tested on different standard data sets and it is observed that it has clear advantage over both manual as well as fully automated heuristic based methods in terms of time and space efficiency.
\end{abstract}

Keywords-2D packing; Crowdsourcing, Hybrid Intelligence approach.

\section{INTRODUCTION}

Human intelligence used in geometric reasoning about shapes, regardless of their educational and social background, is more effective when compared to machine intelligence. Packing problems have its implications in wide industrial applications such as textile, wood, metal, plastics, carbon fiber and glass. In the 2D (two dimensional) packing problems, it is observed that the manual approach as well as the fully automated heuristic-based approaches have their limitations as the manual approach usually takes more time for packing and the fully automated approach achieves limited space efficiency. Further it is found that the amount of research work using both machine and human intelligence together is very much limited which is observed from the state of art review. By combining the human and machine intelligence which is referred as hybrid intelligence in our case, may provide better solution for the 2D packing problem. The hybrid intelligence approach is possible to obtain using the internet crowdsourcing where the outcome of machine intelligence approach for 2D packing may be further refined by a human.

\author{
Jonathan R. Corney\#, G.V. Annamalai Vasantha, A.P. \\ Jagadeesan and A. Lynn \\ Design Manufacture and Engineering Management, \\ University of Strathclyde, Glasgow, UK \\ Email: \#jonathan.corney@strath.ac.uk
}

Crowdsourcing is a process of getting work done generally through internet from crowd of people. Solving 2D packing problems through crowdsourcing, particularly by rural people, will give a greater impact on the industrial applications [12] and also benefit rural people as large population of India live in villages. The packing problem becomes easier when both objects and the containing region are regular/ rectangular in shape, but in most of the practical situations, the irregular shapes have to be used. The geometrical complexity of irregular objects makes the $2 \mathrm{D}$ packing problem a tedious one.

Formally the 2D packing problem can be formulated as follows: Given a regular container $\mathrm{C}$ with set of objects $\mathrm{O}$ containing different types of shapes, find the set of objects $\mathrm{Pi} \in \mathrm{O}$ that can be packed in $\mathrm{C}$ without any overlapping such that the value of efficiency is given by eqn. 1 [1]

$$
\text { Efficiency }=\frac{\sum_{i=0}^{U} \operatorname{Area}\left(P_{i}\right)}{\text { Area }(C)}
$$

The value of efficiency can be maximized by optimization with the help of hybrid intelligence. The main motivation is to apply hybrid intelligence approach on 2D packing problem where the both human intelligence and machine intelligence are utilized to maximize packing efficiency in terms of time and space.

The paper is structured as follows: the related work is discussed in section II and proposed methodology is explained in section III. The results on standard data sets and their analysis are provided in the section IV. Finally the conclusion and future scope of the work are discussed in the section V.

\section{RELATED WORK}

In literature survey, it is found that the previous work is very specific to particular problem domain like, In textile industry the objects generally have similar shapes and sizes. These type of restrictions simplify the problem which are generally found in packing problem domain. It provides motivation to researchers for solving several types of packing problems where the container and objects can be irregular types of shapes. The authors of [2] initially made convex polygon enclosing the given irregular shapes by iterative basing of rectangle on each edge of the given polygon. In 
practical solutions of this type, it will only prove satisfactory if the objects themselves are close to rectangular so that the wasted area is small. The authors of [3] proposed another approach through nesting more than one object together when objects are different from rectangular shape. Another alternative method using rectangle packing is to nest all the objects into identical polygons which can be used to cover the container area. These polygons may include triangles, quadrilaterals, pentagons and hexagons etc. In sstraightforward single pass packing strategies, It uses the pieces of objects in order and place the objects in the container according to given packing rule. It may be repeatable many times for different orderings for best solution and many intelligent method can be used in a single pass. The authors of [4] have sorted the pieces of object in the order of decreasing length and then placed along with two adjacent edges of the stock-sheet. In paper [5], the authors used a random ordering of the pieces with leftmost placement policy. The authors [6] suggested an initial layout packing in Tabu search algorithm.

The authors [7] have used applied genetic algorithms for optimizing the solution. There are several constructive methods which have been used in the literature. The most popular method is the leftmost placement which is used in fixed width of a stock sheet. The authors of [8] and [9] suggested that first feasible bottom-left position can be obtained through the intersection of the nofit polygon and the piece to be placed. The authors of [10] have discussed the power of crowdsourcing in solving the complex 2D packing problem using human intelligence through internet crowdsourcing. The authors of [11] have used crowdsourcing for spatial manufacturing tasks. The authors of [12] have applied the geometrical reasoning task through the crowdsourcing. The authors in [13] and [14] have used combination of human and machine intelligence in the domains of business prediction and classifying celestial bodies respectively. It is observed from above literature review that the use of human intelligence along with machine intelligence has not been utilized in 2D packing domain via crowdsourcing. In our proposed system, a hybrid intelligence system employing both machine and human intelligence is used to solve the $2 \mathrm{D}$ packing problem having irregular objects in computationally and cost effective way. The proposed methodology is detailed in the following section

\section{Proposed Methodology}

It is well known fact that an optimization problem such as $2 \mathrm{D}$ packing is a NP-Hard problem, hence obtaining $100 \%$ efficiency in any optimization task is not a feasible one. In 2D packing task, a greedy algorithm is generally a process that works as recursively to constructs a set of objects from the smallest possible constituent parts. For the optimization of 2D packing problem, a hybrid intelligence approach is used to obtain greater efficiency. The hybrid approach is a combination of machine intelligence and human intelligence. Here, after applying a greedy machine intelligence algorithm, the human intelligence is applied to further refine the solution. In greedy approach, the solution of a given problem basically depends on the solutions to smaller instances of the given problem. Using the greedy algorithm it is easy to understand the solutions for smaller instances.

First, 2D packing task of irregular objects has been implemented using greedy algorithms. Subsequently the user can refine the outcome of the greedy approach based on manual packing. User can drag objects manually using mouse and can place them inside a rectangular bin/container. While dragging and dropping objects user has to ensure that there is no overlapping between two objects and place them in such a way that there is minimal space wastage and hence maximum efficiency gained. In the design of greedy algorithms, several rules were used: largest area first, largest length first, smallest area first, smallest length first etc., and then greedily packing them one by one in this order. While placing the objects inside the bin, any of the following methods may be used: BottomLeft, Next-Fit, First-Fit, and Best-Fit. In the implementation, the Bottom-Left Fill (BLF) technique is employed along with each of above rules for ordering the objects [15].

PROBLEM STATEMENT: Given a finite set of irregular objects $A=\{a 1, a 2, \ldots a n\}$ with associated sizes $Z=\{(x 1$, $\mathrm{y} 1),(\mathrm{x} 2, \mathrm{y} 2), \ldots(\mathrm{xn}, \mathrm{yn})\}$ such that $0<=\mathrm{xi}, \mathrm{yi}<=\mathrm{W}$ where minimal size of a bin is denoted by $\mathrm{W}$ and it place all the objects from $\mathrm{A}$ into the rectangular bin without overlapping with size $\mathrm{X}>=\mathrm{W}, \mathrm{Y}>=\mathrm{W}$ such that the empty space is reduced as lowest as minimum. In the greedy technique, a best solution is basically constructed in some stages. On every step, decision is must because the best possible problem solution based on restrictions. This is not changed later, so every decision should assure feasibility of solution. The greedy algorithm along with the bottom-left fill technique pseudo code is describe below. The greedy technique in 2D bin packing problem based on the dimensions of the object $i$ (its length and height) and the width of the bin. Here first object is placed at null coordinates of the bin. For a position $\mathrm{j}$, for each object is chosen the closer bottom left point (xi, yj) where it can be placed. This point is removed from the list and is inserted two new points: (xj+ lengthj, yj) and (xj, yj+ hightj). If the point is not found in the point's lists, it is necessary to add to the list a point with coordinates 0 and max (height $+y$ ) based on previous object.

- Accomplish the inequality $\mathrm{x}+$ length $<=$ lenght 1 , where the lenght 1 is the length of the first object from the bin.

- The intersection with all previous objects from 1 to $\mathrm{k}$ - 1 need to be null.

In order to prevent some of the already mentioned conditions, it is better to store the points in a sorted list. The BLF procedure is given below[15].

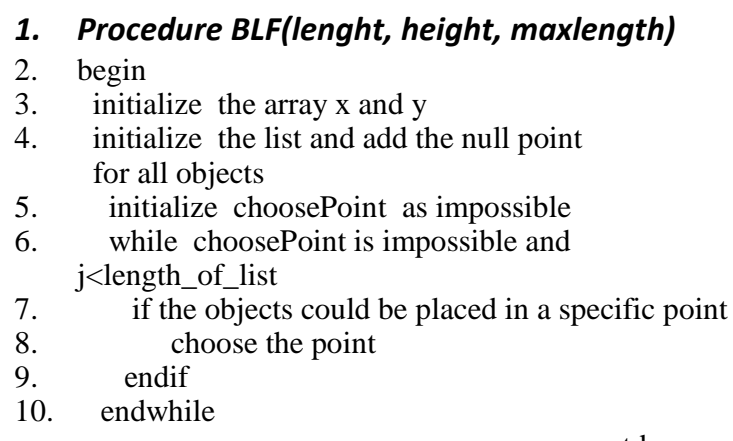

contd... 
11. if choosePoint is possible

12. update the array $\mathrm{x}$ and $\mathrm{y}$

13. remove the point from the position choosePoint from list

14. add the points $\left(\mathrm{x}_{\mathrm{i}}+\right.$ length, $\left.\mathrm{y}_{\mathrm{i}}\right),\left(\mathrm{x}_{\mathrm{i}}, \mathrm{y}_{\mathrm{i}}+\right.$ height $)$ to the points list

15. else

16. if (length $>$ maxlength) the problem has no solution

17. else $x i=0$ and $y i=\max ($ heightk $+y k)$

18. where $\mathrm{k} \in\{1, \ldots \mathrm{i}-1\}$

19. endif

20. endif

21. endfor

22. solutions: the array $x$ and $y$ with $\left({ }_{x i}, y_{i}\right)-$ the coordinates of object $\mathrm{i}$.

23. end

2D packing of irregular objects has been categorized into 2 parts.

- Manual packing of objects

- Semi-automated (hybrid approach) packing of objects

DATA SETS USED: We have used 2 data sets ALBANO [16] and DAGLI [17], but our algorithm can be generalized for other data sets also.

ALBANO DATA SET: comprises of 24 objects with 8 different shapes and their replicas.

DAGLI DATA SET: comprises of 30 objects with different shapes and their replicas.

CREATION OF SHAPES: We created shapes using kineticjs library in php with the help of coordinates stored in an XML file.

COLLISION DETECTION: In order to avoid the overlapping of objects, collision detection has been performed where the colour of the shapes, which are overlapping, changes.

EFFICIENCY AND LENGTH USED: As the user places each objects in the bin, each time the length and the efficiency gets updated.

ROTATION OF OBJECTS: In order to avoid space wastage, the user can use the nudge and rotate keys to rotate the objects. TIMER: A timer is running on every page which calculates the time spent on packing.

\section{RESUlTS \& ANALYSIS}

The user interface for the proposed hybrid approach is depicted in figure.1. User have to select the type by clicking on the corresponding action button provided in the interface. The manual packing for using the proposed software is depicted in figure 2(a) and 2(b) on Albano data. User can manually drag objects using mouse and place them in the rectangular bin. While dragging and dropping objects user has to ensure that there is no overlapping between two objects and place them in such a way that there is minimal space wastage and maximum efficiency gained.

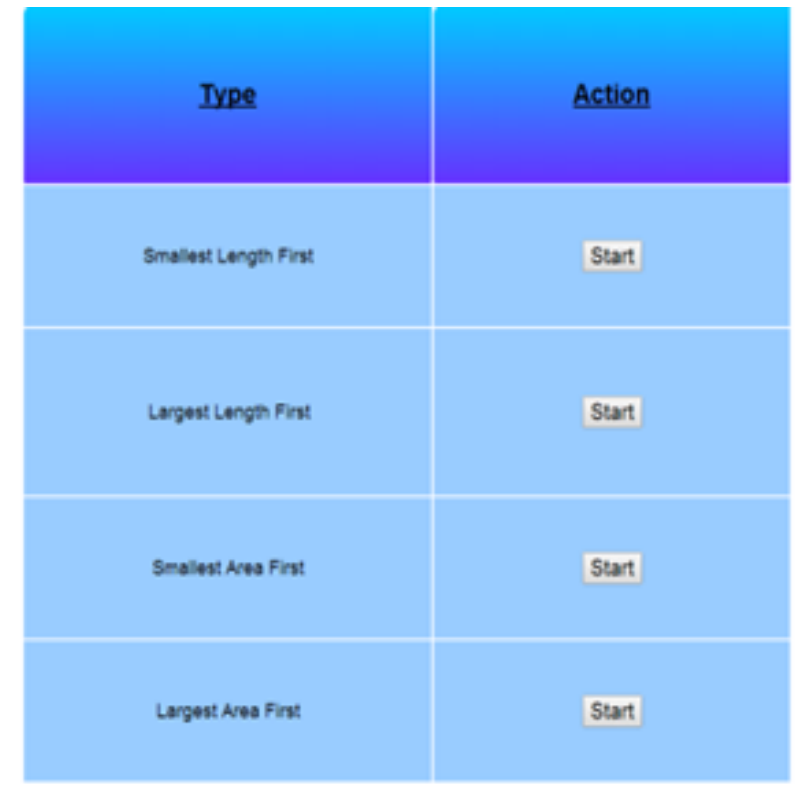

Fig.1. User Interface of the Proposed System

For semi-automated packing of objects, greedy algorithms has been used in order to find the order in which the objects are to be placed in the bin. Several types like largest length first, smallest area first etc. have been used. User can select any of the type to find the maximum efficiency that can be obtained using greedy algorithm. Even in semi-automated packing of objects, user can change the position of objects, rotate the objects, detect collision etc. to maximize efficiency (similar to the manual approach). The experiments on different types of criteria used in greedy algorithm of the proposed method on Albano data are depicted in the Figure.2 (c), (d), (e), and (f).

The results of manual and semi-automated packing task which are conducted on 20 participant in the form of efficiency (in \%) and time (in sec) where the number of participant: 20 students, Gender: 15 male, 05 female, Education: $10+2$ examination, Average age: 21 years, Experience: Nil, Proficient in computer knowledge/ skill. The datasets we have used are Albano and Dagli datasets $[15,16]$. The largest length first criteria have been used in the proposed semi-automated approach. The result are shown in the TABLE 1 and TABLE 2.

TABLE 1. Results of Manual and Semi-automated 2D Packing Task in the form of efficiency (in \%) on 20 participants

\begin{tabular}{|c|l|l|l|l|}
\hline Participants & $\begin{array}{l}\text { Efficiency } \\
\text { of } \\
\text { Albano } \\
\text { Manual } \\
\text { packing } \\
\text { (in \%) }\end{array}$ & $\begin{array}{l}\text { Efficiency } \\
\text { of Albano } \\
\text { Semi- } \\
\text { automated } \\
\text { packing } \\
\text { (in \%) }\end{array}$ & $\begin{array}{l}\text { Efficiency } \\
\text { of Dagli } \\
\text { Manual } \\
\text { packing } \\
\text { (in \%) }\end{array}$ & $\begin{array}{l}\text { Efficiency } \\
\text { of Dagli } \\
\text { Semi- } \\
\text { automated } \\
\text { packing } \\
\text { (in \%) }\end{array}$ \\
\hline 1. & 82.39 & 68.35 & 79.71 & 83.02 \\
\hline 2. & 81.02 & 81.46 & 77.30 & 72.89 \\
\hline 3. & 73.78 & 86.19 & 72.68 & 85.15 \\
\hline 4. & 79.29 & 84.74 & 66.02 & 66.09 \\
\hline 5. & 77.18 & 78.85 & 77.32 & 69.66 \\
\hline 6. & 81.50 & 78.01 & 73.15 & 76.20 \\
\hline 7. & 80.76 & 78.85 & 76.80 & 68.47 \\
\hline
\end{tabular}




\begin{tabular}{|l|l|l|l|l|}
\hline 8. & 79.00 & 85.35 & 66.02 & 68.50 \\
\hline 9. & 71.01 & 85.96 & 71.60 & 78.54 \\
\hline 10. & 69.92 & 78.01 & 64.96 & 77.34 \\
\hline 11. & 79.03 & 78.71 & 68.18 & 68.12 \\
\hline 12. & 75.70 & 79.29 & 69.17 & 68.70 \\
\hline 13. & 81.06 & 79.57 & 70.12 & 76.23 \\
\hline 14. & 84.05 & 81.21 & 73.93 & 76.67 \\
\hline 15. & 79.00 & 79.84 & 79.75 & 76.96 \\
\hline 16. & 71.18 & 78.43 & 73.41 & 65.39 \\
\hline 17. & 73.32 & 76.18 & 78.91 & 72.28 \\
\hline 18. & 80.05 & 80.54 & 80.02 & 82.02 \\
\hline 19. & 78.61 & 80.14 & 78.00 & 81.02 \\
\hline 20. & 78.06 & 78.27 & 79.60 & 79.94 \\
\hline
\end{tabular}

In the table1, the results of manual and semi-automated packing task are shown in the form of efficiency (in \%) on albano and dagli datasets. The average result of albano manual packing efficiency is $77.80 \%$ and dagli manual packing efficiency $73.83 \%$ whereas the average result of albano semiautomated packing task is $79.90 \%$ and the average result of dagli is $74.66 \%$. So from the above table, the results of semiautomated packing on albano and dagli datasets are better than the manual packing of albano and dagli datasets.

Timer is runing:

27

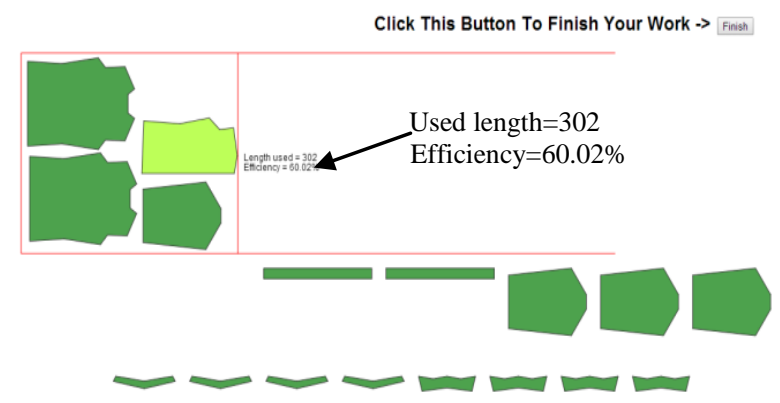

Fig.2. (a).Efficiency and length updated each time an object is placed
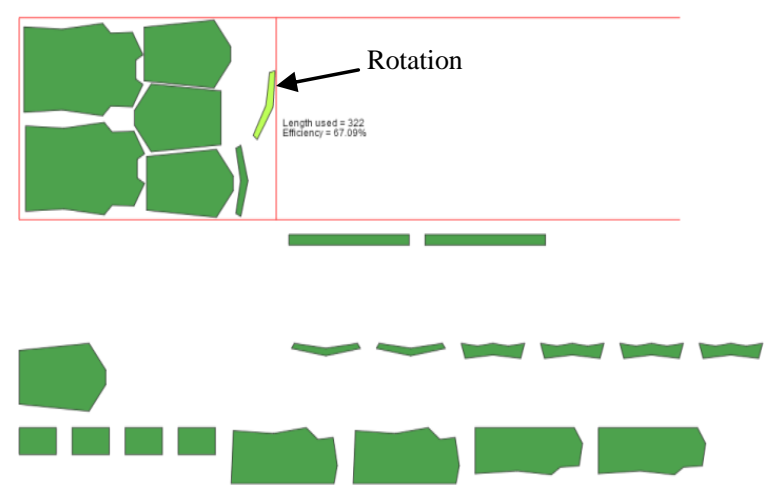

Fig.2. (b). Rotation of objects

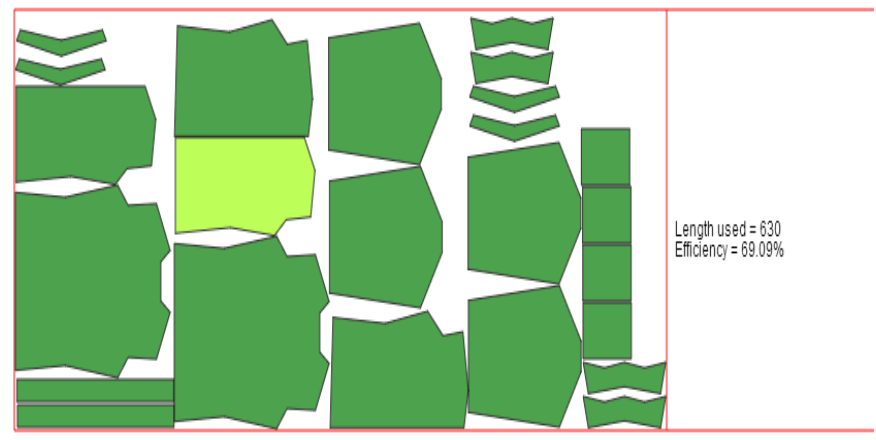

Fig. 2. (c). Largest length first ordering, Efficiency achieved $=69.09 \%$

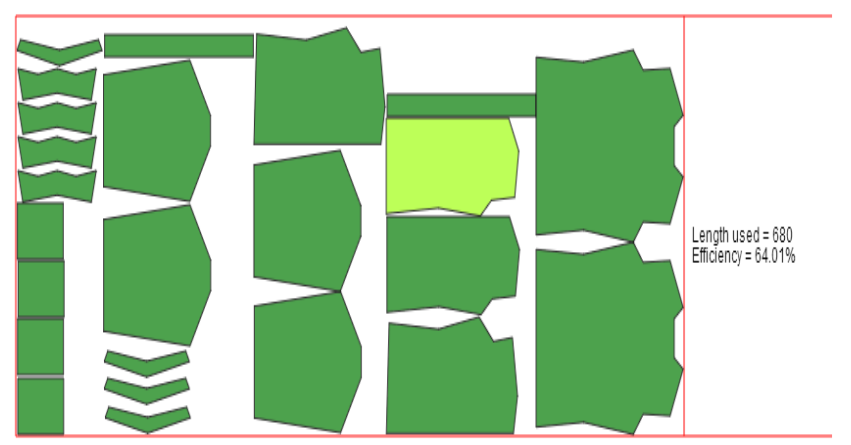

Fig.2. (d). Smallest length first ordering, Efficiency achieved $=64.01 \%$

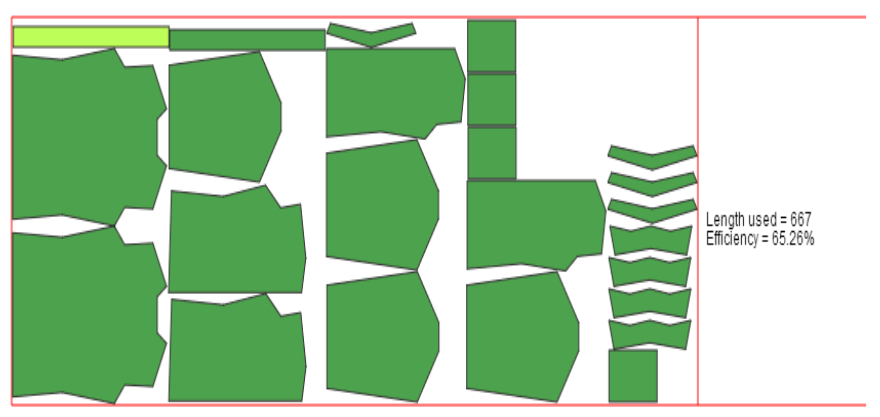

Fig.2. (e). Largest area first ordering Efficiency achieved $=65.26 \%$

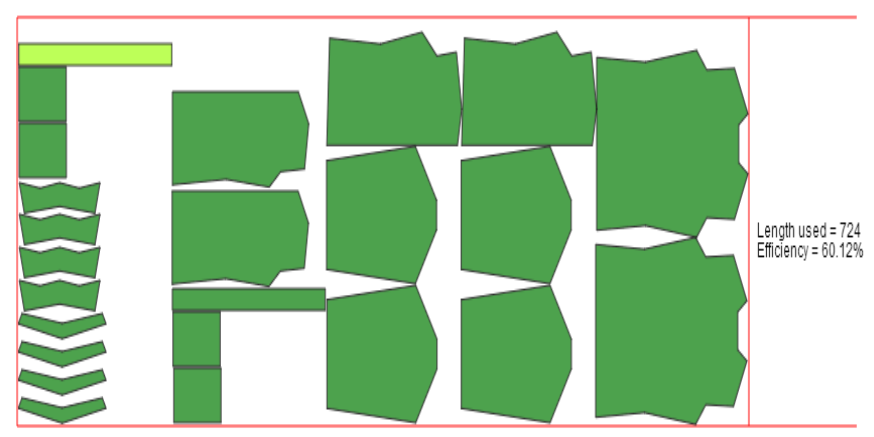

Fig.2. (f). Smallest area first ordering, Efficiency achieved $=60.12 \%$ 
Fig. 2. (a) (b) Results of Manual packing method. (c), (d), (e), (f) Results obtained using Greedy approach with different criteria.

TABLE 2. Results of Manual and Semi-automated 2D Packing in the form of time(in sec) on 20 participants

\begin{tabular}{|c|c|c|c|c|}
\hline Participants & $\begin{array}{c}\text { Time } \\
\text { Taken in } \\
\text { Albano } \\
\text { Manual } \\
\text { packing } \\
\text { (in sec) }\end{array}$ & $\begin{array}{l}\text { Time Taken } \\
\text { in Albano } \\
\text { Semi- } \\
\text { automated } \\
\text { Packing (in } \\
\text { sec) }\end{array}$ & $\begin{array}{c}\text { Time } \\
\text { Taken in } \\
\text { Dagli } \\
\text { Manual } \\
\text { packing } \\
\text { (in sec) }\end{array}$ & $\begin{array}{c}\text { Time } \\
\text { Taken in } \\
\text { Dagli } \\
\text { Semi- } \\
\text { automated } \\
\text { Packing } \\
\text { (in sec) }\end{array}$ \\
\hline 1. & 227 & 946 & 656 & 238 \\
\hline 2. & 1240 & 230 & 820 & 911 \\
\hline 3. & 728 & 438 & 303 & 731 \\
\hline 4. & 619 & 185 & 219 & 199 \\
\hline 5. & 1638 & 905 & 905 & 212 \\
\hline 6. & 729 & 189 & 977 & 268 \\
\hline 7. & 827 & 160 & 427 & 125 \\
\hline 8. & 666 & 656 & 1214 & 271 \\
\hline 9. & 915 & 777 & 1325 & 287 \\
\hline 10. & 778 & 189 & 1093 & 178 \\
\hline 11. & 2320 & 733 & 1843 & 118 \\
\hline 12. & 578 & 287 & 1103 & 346 \\
\hline 13. & 1091 & 536 & 906 & 260 \\
\hline 14. & 672 & 557 & 1081 & 259 \\
\hline 15. & 1892 & 1087 & 1087 & 458 \\
\hline 16. & 790 & 262 & 860 & 394 \\
\hline 17. & 411 & 228 & 557 & 288 \\
\hline 18. & 457 & 290 & 543 & 350 \\
\hline 19. & 577 & 479 & 760 & 634 \\
\hline 20. & 915 & 778 & 302 & 287 \\
\hline
\end{tabular}

In above table2, the results of manual and semi-automated packing task are shown in the form of time (in sec.) on albano and dagli datasets. The average result of albano manual packing the time taken is $903.5 \mathrm{sec}$. and dagli manual packing the time taken is $849.05 \mathrm{sec}$. whereas the average result of albano semi-automated packing the time taken is $495.6 \mathrm{sec}$. and the average result of dagli the time taken is $340.7 \mathrm{sec}$. So from the above table, the results of time taken by semiautomated packing on albano and dagli datasets are much better than the time taken by manual packing of albano and dagli datasets. The tabular results are also plotted in graphical forms in figures 3, 4, 5 and 6. Paired t-test reveals that there is a statistically significant difference in time taken between the manual and the semi-automated approach for solving both Albano (t19=3.945, $\mathrm{p}=.001)$ and Dagli $(\mathrm{t} 19=4.694, \mathrm{p}<.0001)$ $2 \mathrm{D}$ packing problems. The average time taken shows that the semi-automated approach is much quicker than the manual approach. At the same time, the semi-automated approach retains the benefit of space efficiency of manual approach over the fully automated packing.
TABLE 3. Descriptive statistics of TABLE 1 and TABLE 2

\begin{tabular}{|c|c|c|c|c|}
\hline \multicolumn{5}{|c|}{ Descriptive Statistics } \\
\hline $\mathbf{N}=\mathbf{2 0}$ & Minimum & Maximum & Mean & $\begin{array}{c}\text { Std. } \\
\text { Deviatio } \\
\text { n } \\
\end{array}$ \\
\hline $\begin{array}{l}\text { AlbanoManualEfficiency } \\
(\%)\end{array}$ & 69.92 & 84.05 & 77.80 & 4.03 \\
\hline $\begin{array}{l}\text { AlbanoSemi- } \\
\text { automatedEfficiency }(\%)\end{array}$ & 68.35 & 86.19 & 79.90 & 3.96 \\
\hline $\begin{array}{l}\text { AlbanoManualTimetaken } \\
\text { (Seconds) }\end{array}$ & 227 & 2320 & 903.50 & 516.60 \\
\hline $\begin{array}{l}\text { AlbanoSemi- } \\
\text { automatedTimeTaken } \\
\text { (Seconds) }\end{array}$ & 160.00 & 1087.00 & 495.60 & 294.68 \\
\hline $\begin{array}{l}\text { DagliManualEfficiency } \\
(\%)\end{array}$ & 64.96 & 80.02 & 73.83 & 5.09 \\
\hline $\begin{array}{l}\text { DagliSemi- } \\
\text { automatedEfficiency }(\%)\end{array}$ & 65.39 & 85.15 & 74.66 & 6.01 \\
\hline $\begin{array}{l}\text { DagliManualTimetaken } \\
\text { (Seconds) }\end{array}$ & 219 & 1843 & 849.05 & 397.52 \\
\hline $\begin{array}{l}\text { DagliSemi- } \\
\text { automatedTimeTaken } \\
\text { (Seconds) }\end{array}$ & 118.00 & 911.00 & 340.70 & 202.87 \\
\hline
\end{tabular}

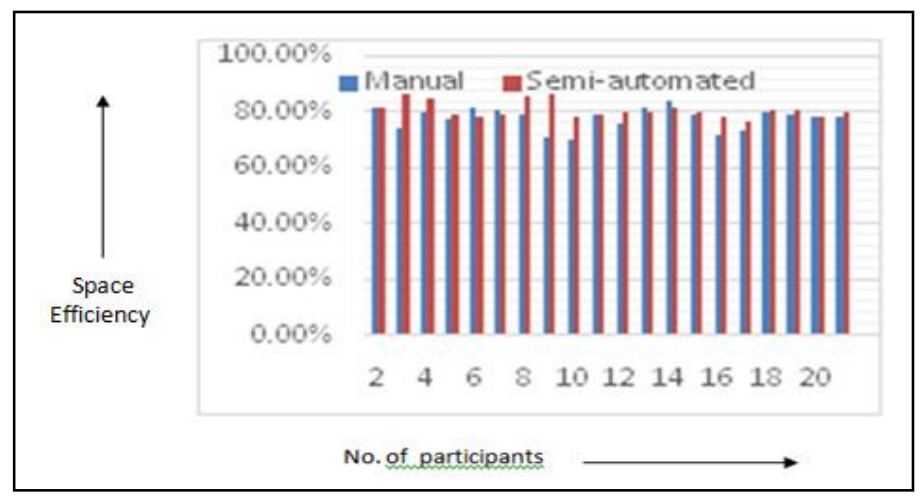

Fig. 3. Graphical representation of the Results of Manual and Semi-automated 2D Packing Task on Albano datasets in the form of efficiency (in \%) on 20 participants.

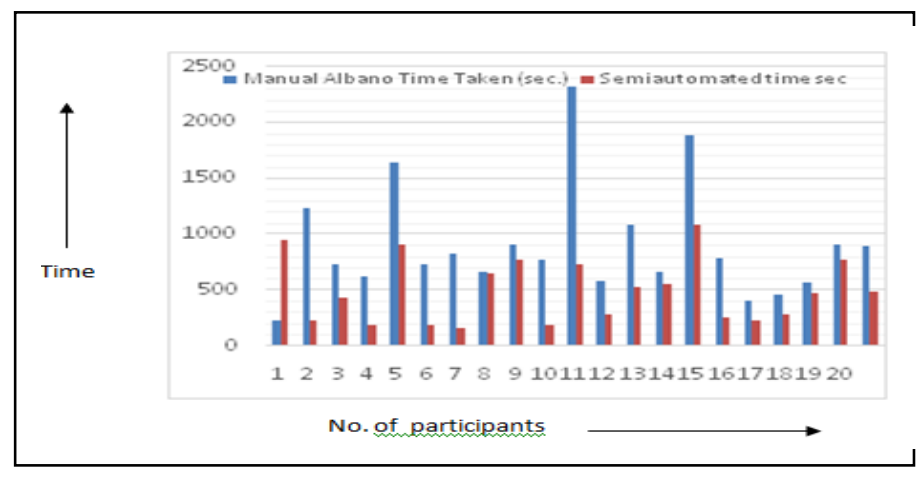

Fig. 4. Graphical representation of the Results of time taken by Manual and Semi-automated 2D Packing Task on Albano datasets on 20 participants.

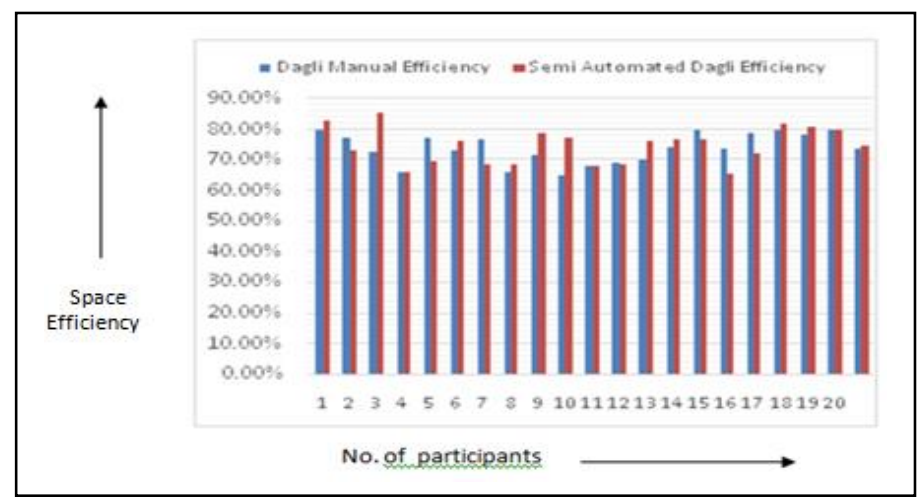


Fig. 5. Graphical representation of the Results of Manual and Semi-automated 2D Packing Task on Dagli datasets in the form of efficiency (in \%) on 20 participants.

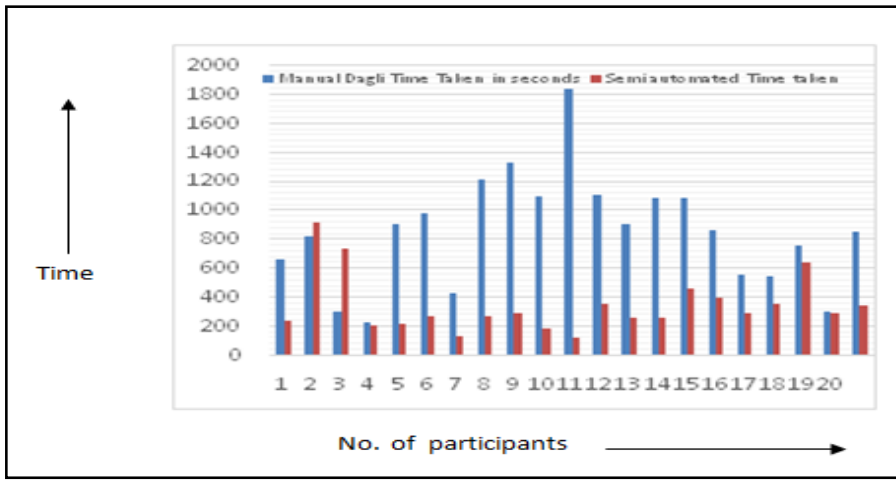

Fig. 6. Graphical representation of the Results of time taken by Manua and Semi-automated 2D Packing Task on Dagli datasets on 20 participants.

Also we have applied hybrid intelligence based 2D Packing on Rural BPO Workers in India and we have got the feasible results which are comparable from previous results.

\section{CONCLUSIONS \& FUTURE WORK}

A hybrid intelligence based semi-automated approach combining human and machine intelligence has been proposed to solve the $2 \mathrm{D}$ packing problem having irregular shape objects. The greedy technique is using Bottom-Left-Fill (BLF) procedure, involving iterative placement of objects from the lower left corner of the bin/container. Each object is placed as low as possible such that it does not overlap with other objects. The greedy algorithm, in the proposed semiautomated packing of objects, represents the problem as an ordered list of pieces to be packed where the order is decided by a placement heuristic. Several packing arrangements involving greedy algorithm have been used and we have applied manual 2D packing and semi-automated 2D packing methods on computer skilled $10+2$ passed students through internet crowdsourcing. We have got the effective results, particularly in terms time efficiency, with proposed semiautomated packing system over the manual packing system. At the same time, the semi-automated approach retains the benefit of space efficiency of manual approach over the fully automated packing which has been verified on various subjects including rural people. In Future, the authors wish to extend the hybrid intelligence approach for 3D Packing problems through internet crowdsourcing.

\section{ACKNOWLEDGEMENT}

This research is collaboratively funded by the DST, India and the EPSRC, UK as part of the research project "Distributing Industrial Optimization Tasks To Rural Worker".

\section{References}

[1] Ping Chen,Zaohui Fu,Andreq Lim and Brain Rodrigues,"The TwoDimensional Packing Problem For Irregular Objects," International Journal on Artificial Intelligence Tools, Vol. 13, Issue 3, 2004, pp. 429448.

[2] H. Freeman and R. Shapira, "Determining the minimum area encasing rectangle for an arbitrary closed curve," Communications of the ACM, Vol. 18, Issue 7, July 1975, pp. 409-413.

[3] M. Adamowica and A. Albano, "Nesting two dimensional shapes in rectangular modules," Computer Aided Design, 8(1), Jan 1976, pp. 27 33 .

[4] W. Qu and J. Sanders, "A nesting algorithm for irregular parts and factors affecting trim losses," International Journal of Production Research, Vov. 25, Issue 3, 1987, pp. 381-397.

[5] K. A. Dowsland and W. Dowsland, "Heuristic approaches to irregular cutting problems," Working Paper EBMS/13, European Business Management School, UC Swansea, UK, 1993.

[6] Blazewicz, J., Hawryluk, P. and Walkowiak, R., "Using a tabu search approach for solving the two-dimensional irregular cutting problem," Annals of Operations Research, 41, 1993, pp. 313-327.

[7] Sakait Jain and Hae Chang Gea."Two-Dimensional Packing Problem Using Genetic Algorithms," Engineering with computers, 1998, pp. 14: 206-213.

[8] Kathryn A. Dowsland, Subodh Vaid and William B. Dowsland, "An algorithm for polygon placement using a bottom-left strategy," European Journal of Operational Research, Vol. 141, Issue 2, 2002, pp. 371-381.

[9] M. Teresa Costa, A. Miguel Gomes and Jose' F. Oliveira, "Heuristic approaches to large-scale periodic packing of irregular shapes on a rectangular sheet," European Journal of Operational Research, 192, 2009, pp. 29-40.

[10] Corney, Jonathan, Gokula Annamalai Vasantha, Andrew Lynn, Ananda Jagadeesan, Nuran Acur, Marisa Smith, and Anupam Agarwal, "Computing the Incomputable with Human Processing Units," In Intelligent Interactive Technologies and Multimedia, Springer Berlin Heidelberg, 2013, pp. 14-24.

[11] Vasantha, Annamalai, Gokula Vijayumar, Jonathan Corney, Ananda Prasanna Jagadeesan, Andrew Lynn, Nuran Acur Bakir, Marisa Smith, and Anupam Agrawal. "Towards crowdsourcing spatial manufacturing tasks from rural India." In 12th International Conference on Manufacturing Research, 2014, pp. 91-96.

[12] Jagadeesan, A. Prasanna, A. Lynn, Jonathan R. Corney, X. T. Yan, Jan Wenzel, Andrew Sherlock, and W. Regli, "Geometric reasoning via internet crowdsourcing," In 2009 SIAM/ACM Joint Conference on Geometric and Physical Modeling, 2009, pp. 313-318.

[13] Yiftach Nagar and Thomas Malone, "Making Business Predictions by Combining Human and Machine Intelligence in Prediction Markets," Proceedings of ICIS 2011, Shanghai, China, December 5, 2011.

[14] Ece Kamar, Severin Hacker and Eric Horvitz, "Combining human and machine intelligence in large-scale crowdsourcing," Proceedings of the 11th International Conference on Autonomous Agents and Multiagent Systems (AAMAS'12), Vol. 1, 2012, pp. 467-474.

[15] Camelia-M. Pintea, Cristian Pascan and Mara Hajdu-Măcelaru, "Comparing several heuristics for a packing problem," Int. J. of Advanced Intelligence Paradigms, Vol.4, No.3/4, 2012, pp. 268 - 277.

[16] Albano, A. and Sappupo, G., "Optimal allocation of two-dimensional irregular shapes using heuristic search methods," IEEE Transactions on Systems, Man and Cybernetics, SMC-10, 1980, pp. 242-248.

[17] Ratanapan, K. and Dagli, C.H., "An object-based evolutionary algorithm for solving irregular nesting problems," In: Proceedings for Artificial Neural Networks in Engineering Conference (ANNIE'97), vol.7, ASME Press, New York, 1997, pp. 383-388. 